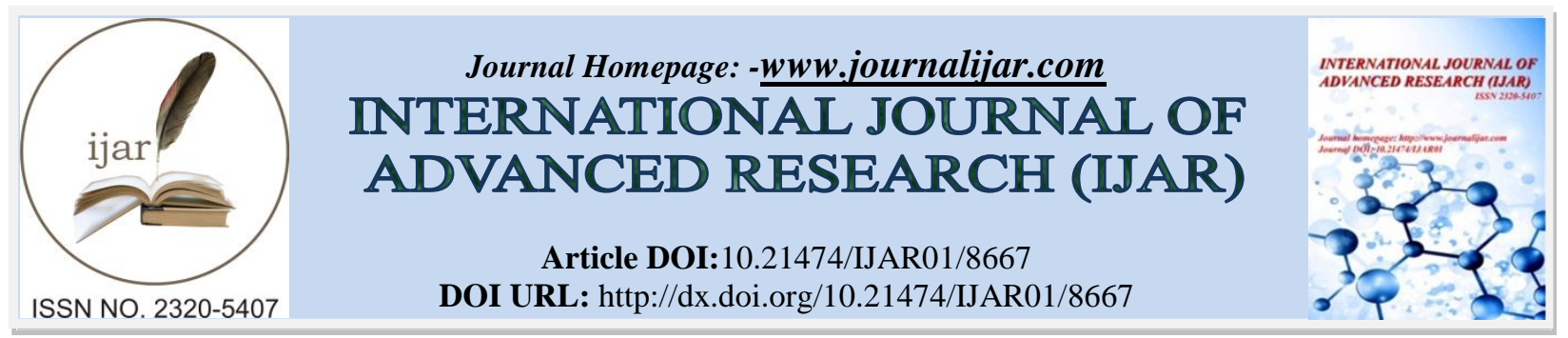

RESEARCH ARTICLE

\title{
VERRUCOUS CARCINOMA OF BUCCAL MUCOSA: A CASE REPORT AND REVIEW.
}

Sahil Kohli ${ }^{1}$ and Christopher Vinay Shinde ${ }^{2}$.

1. Senior Lecturer, Department of Oral Medicine and Radiology, RKDF Dental College and Research Centre, Bhopal, MP, India.

2. Senior Lecturer, Department of Oral Medicine and Radiology, Pacific Dental College and Research Centre, Udaipur, Rajasthan, India.

\section{Manuscript Info}

Manuscript History

Received: 11 January 2019

Final Accepted: 13 February 2019

Published: March 2019

\section{Abstract}

Copy Right, IJAR, 2019,. All rights reserved.

\section{Introduction:-}

Verrucous carcinoma is a lesion with minimum aggressive potential. It is a variant of squamous cell carcinoma. It most commonly affects oral cavity with buccal mucosa being the commonest site affected. ${ }^{1}$ It presents predominantly as an exophytic growth with a pebbly micronodular surface and a slow growing rate. ${ }^{2}$ The anatomy of buccal mucosa and buccal space allows for extension of carcinoma unimpeded by any anatomic barriers to neighbouring intraoral subsites and structures. ${ }^{3}$ Tobacco,alcohol and opportunist viral infections are the most associated etiologies with verrucous carcinoma. ${ }^{1}$ Ackerman first recognised this lesion as a distinct entity in 1948, so it is also known as ACKERMAN'S TUMOUR. ${ }^{5}$ The diagnosis in verrucous lesions of the oral cavity can range from verrucous hyperplasia to verrucous proliferative leukoplakia and verrucous carcinoma. ${ }^{6}$

\section{Case report:}

A 48 year old male patient reported with a chief complain of assymetry on the left side of face since 3 months (Figure $1 \mathrm{a}, \mathrm{b}$ ). He initially observed ulceration on the inner side of left cheek but did not took any medication for the same. He also observed extraoral swelling on left side of face which gradually increased in size. He developed pain 3 months back which was initially mild and intermittent but has aggrevated since 7 days. He has a history of chewing supari since 40 years, 15 times a day in lower left buccal vestibule for 5 minutes following which he used to spit out contents. He is suffering from mild bronchitis and mild cardiomegaly, according to diagnostic reports of a government hospital. There was no relevant family history. On general examination, he had normal gait and posture and was well oriented, consious and moderatly built. No evidence of pallor, icterus, cyanosis and clubbing was present. Single left submandibular lymph node was palpable of approximately $1 \mathrm{~cm}$ by $1 \mathrm{~cm}$ in size, ovoid in shape, non-tender, fixed on palpation and firm in consistency.

During intraoral examination,oninspection,there was presence of solitary proliferative verrucous growth on left buccal mucosa extending anteroposteriorly in relation to mesial side of 34 uptoretromolar area and superioinferiorly from level of occlusal plane upto depth of buccal vestibule in relation to $34,35,36,37$. The lesion was approximately $30 \mathrm{~mm}$ by $20 \mathrm{~mm}$ in size,well-defined with irregular margins. The lesion appeared exophytic and cauliflower shaped (Figure 2).Surface of the lesion was irregular with rough consistency and colour of the lesion varied from pink in periphery to white in the centre.Onpalpation,inspectory findings of size,site, surface and shape were confirmed.The lesion was non-scrappable,non-tender,andindurated.There was no bleeding on touching.Hard tissue examination 
revealed pus discharge in relation to 36,37 and grade III mobility in relation to 37 was present with poor oral hygiene.

During extraoralexamination,swelling was present on left side of face, $50 \mathrm{~mm}$ by $60 \mathrm{~mm}$ in size,oval in shape and extends anteroposteriorly from left corner of mouth upto middle third of ear and superioinferiorly from alatragal line upto lower border of mandible.Thecolour was normal and consistency firm. The borders were diffused and there was no compressibility/reducibility.The swelling was non-tender on palpation and temperature was afebrile.Theinterincisal mouth opening was $40 \mathrm{~mm}$.

Based on the clinical examination,a provisional diagnosis of verrucous carcinoma of left buccal mucosa was given.Differential diagnosis of verrucousleukoplakia,verrucous hyperplasia was given. Complete blood count and random blood sugar test revealed no abnormality,so incisional biopsy was suggested.

\section{Radiographic features:}

Orthopantomograph revealed a well-defined radiolucency on left side of mandible,extendinganteroposteriorly from distal side of 37 upto ramus of mandible and superoinferiorly from the level of occlusal plane extending slightly above the left inferior alvelolar nerve canal. The shape is roughly oval and internal structure is completely radiolucent (Figure 3).

\section{Histopathology report:}

$\mathrm{H}$ and $\mathrm{E}$ section reveal hyperplastic and parakeratotic epithelium with heaped up large papillae showing mild to moderate dysplasia. There are few superficial nests of atypical cells with intact basement membrane.

\section{Histopathological diagnosis:}

Overall features suggestive of Verrucous carcinoma.

\section{Discussion:-}

Oral verrucous carcinoma most commonly affects elderly males with adverse habits of tobacco and alcohol. ${ }^{1}$ Chemical carcinogens have long been implicated in the etiology of this lesion but identification of a specific etiological factor is often difficult because of incidence of multiple habits. The habitual chewing of "paan", a mixture of betel leaf,lime,betel nuts and tobacco,have been extensively implicated for the high incidence of verrucous type of oral cancers in India. ${ }^{7}$ More recently,studies have confirmed the association between Human papilloma virus(HPV) and verrucous carcinoma by detecting HPV-DNA types 6,11,16 and 18 by polymerase chain reaction, restriction fragment analysis and DNA slot-blot Hybridization. ${ }^{13}$ Verrucous carcinoma presents predominantly as an exophytic growth with a pebbly,micronodularsurface, a slow growing rate, and becomes locally invasive if not treated properly. ${ }^{2}$ It is a unique clinicopathological variant of squamous cell carcinoma,occuring mainly in oral cavity and larynx,buccal mucosa being most commonly involved.It is a very rare entity first described by Ackerman in 1948. Various synonyms are used to describe this tumour,including Ackerman's tumour,BuschkeLoewensteintumour,florid oral papillomatosis,epitheliomacuniculatum,and carcinoma cuniculatum. ${ }^{10}$ Oral cancer is the sixth most common cancer worldwide and squamous cell carcinoma accounts for $90 \%$ of these cases. Oral cancer is definitely a preventable condition,due to possibility of early detection and prompt treatment. However, majority of cases are detected at a much advanced stage leading to poor prognosis and low survival rate of patients. ${ }^{4}$

The macroscopic appearance of Ackerman's tumour depends on several factors like duration of lesion,degree of keratinisation,and the changes in adjacent mucosa.The fully developed carcinoma is rough,shaggy with papillomatoussurface.The surface is usually heavily keratinised.The presence of keratin on an irregular moist mucosal surface gives the lesion its white, warty clinical appearance. ${ }^{5}$ It presents with hyperplastic epithelium with abundant keratin superficially projecting as exophytic church-spire keratosis and also depicting parakeratin plugging ,which is believed to be characteristic of this tumour. ${ }^{8}$ Typically,but not invariably,there is an associated mononuclear inflammatory reaction in the stroma immediately adjacent to advancing margin. The inflammatory cells are usually plasma cells and lymphocytes but giant cell reaction to extruded keratin is also commonly encountered. ${ }^{11}$ The bulbous well-oriented reteridges show endophytic growth pattern with pushing borders. Abrupt transition from normal epithelium to endophytic ingrowth is taken as an important parameter to differentiate it from benign verrucous growths. ${ }^{8}$ Distinction from classical squamous cell carcinoma is a frequent problem for clinicians because of the extensive nature of the lesion mimicking an invasive cancer. An important help could be offered by 
molecular approaches.Verrucous carcinoma shows the characteristics of cell kinetics that are similar to those of normal epithelium and not to conventional squamous cell carcinoma. S-phase is confined to basal layer , unlike the invasive cancer.By flow cytometry,verrucous carcinoma is a diploid lesion,on the contrary,the conventional squamous cell carcinoma often shows aneuploidy and genomic instability. ${ }^{9}$ Although the adjacent cervical lymph nodes are often enlarged, regional and distant metastases rarely occur in this tumour. ${ }^{7}$

Verrucous squamous cell carcinoma is probably one of the most difficult and problematic lesions to diagnose in almost every instance.This is because the lesion is not cytologically malignant and therefore evidence of invasion is required for definite diagnosis. ${ }^{15}$ Multiple deep biopsies are recommended to avoid the problem of underdiagnosis. ${ }^{13}$ With the knowledge of risk factors,primary prevention through the elimination of tobacco consumption,the moderation of alcohol intake and chemoprevention is urgently needed.Research on the natural history of the disease,particularly which precancers will progress over time, would help to develop screening programmes tailored more to the individual disorders.Development of tumour markers with high sensitivity and specificity could assist the detection of patients and lesions at risk. ${ }^{14}$ Because metastases is an extremely rare event in verrucouscarcinoma,the treatment of choice is surgical excision without radical neck dissection.Recently successful treatment of an extensive verrucous carcinoma with intra-arterial infusion of methotrexate or topical 5aminolevulinic acid mediated photodyanamic therapy was reported. ${ }^{13}$ Because of reported incidence of anaplastic transformation following radiotherapy,manycentres recommend wide field surgical resection with good oncoclearance as preferred treatment modality. ${ }^{12}$ The use of buccal fat pad has increased in popularity because of its reliability,ease of harvest,and low complication rate.It has been used as a pedicle or free graft in reconstructing small to medium sized defects intraorally. ${ }^{10}$

\section{Conclusion:-}

Verrucous lesions of the oral cavity are a distinct clinical entity with varied histopathology. ${ }^{6}$ In most of the cases verrucouscarcinoma,verrucoushyperplasia,verrucous keratosis are clinically indistinguishable from each other so histopathological evidence is necessary to give an appropriate diagnosis. ${ }^{1}$ An adequate full thickness biopsy specimen must be taken when a clinician suspects a verrucous carcinoma and multiple biopsies may be needed to rule out a conventional squamous cell component in verrucous carcinoma. ${ }^{5}$ The prognosis of verrucous carcinoma is better than other kinds of life-threatening malignant tumours.Various treatment modalities include surgery,chemotherapy,radiation or combination of these and photodyanamic therapy which has been recently reported. ${ }^{10}$

Figure 1a,b:-Frontal And Lateral View Of The Patient.
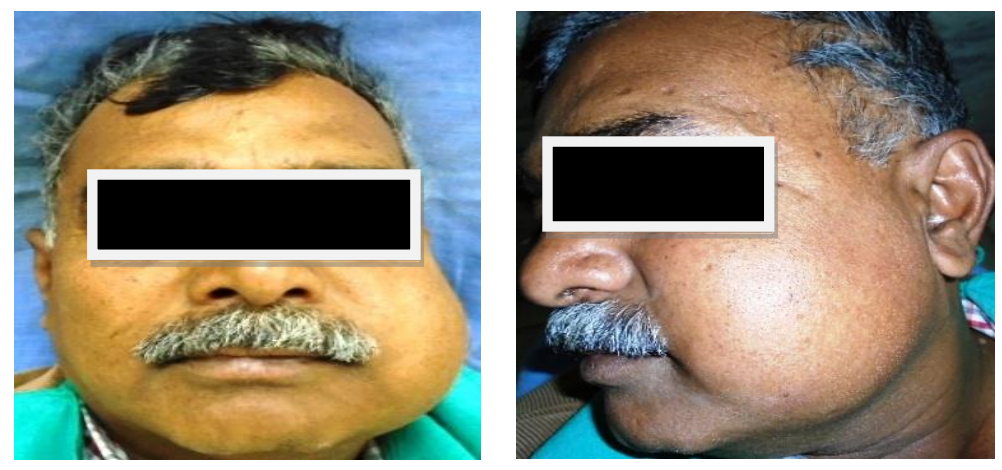

Figure 2:-Verrucous Growth Of Left Buccal Mucosa.

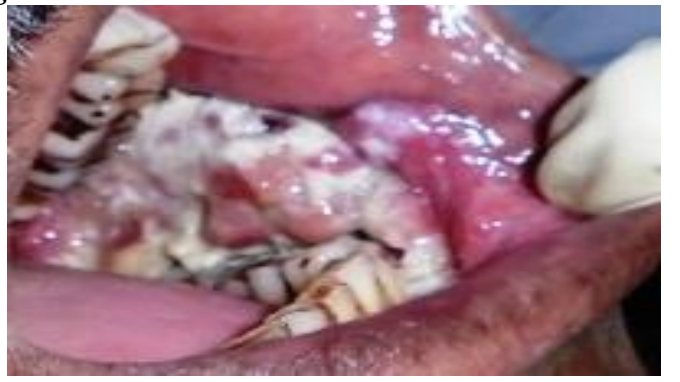


Figure 3:-OrthopantomographOf The Patient.

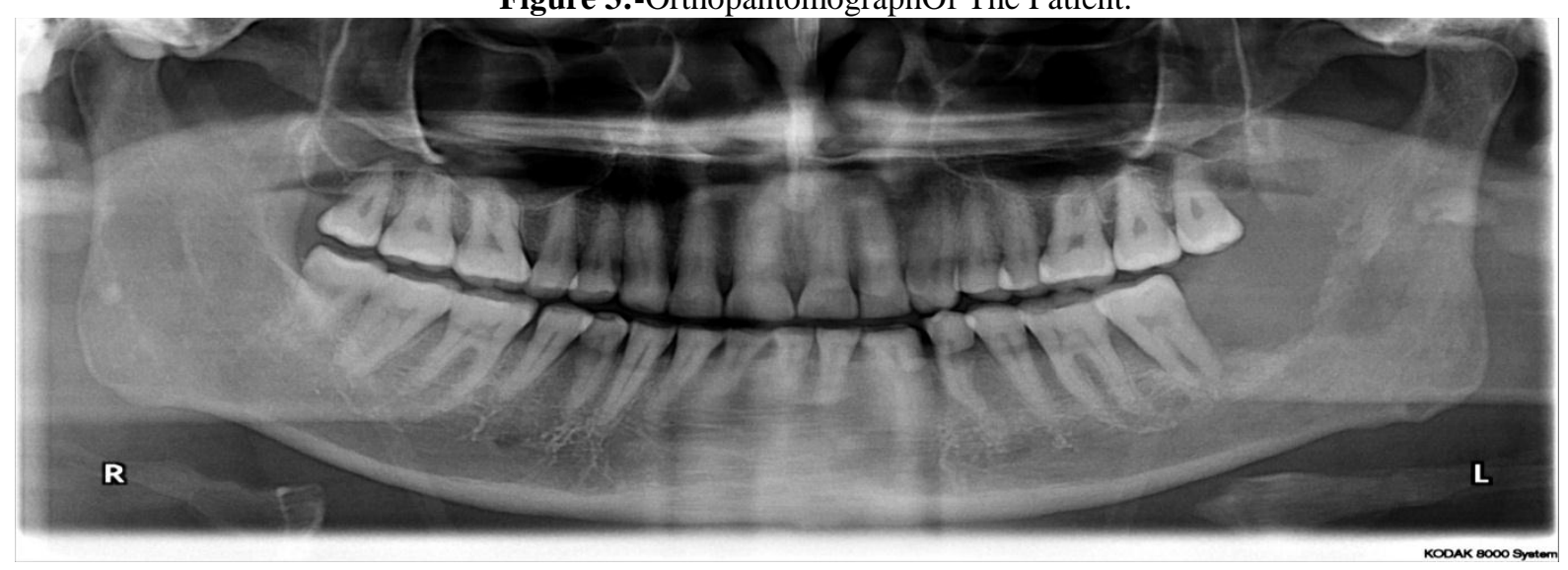

\section{References:-}

1. AshaML,ViniK,ChatterjeeI,PatilP.Verrucous Carcinoma of BuccalMucosa:A Case Report.Int J Adv Health Sci 2014; 1(4):19-23

2. RaoDS,KalappanavarAN,AliIM,AnnigeriRG.Verrucous carcinoma $\quad$-an enigma:Case report and review.ContempClin Dent 2016;7:391-3

3. De Conde A et al.Squamous cell carcinoma of buccalmucosa:a 40 year review.Am J Otolaryngol 2012;33:673677

4. GangavatiR,BaadR,VibhuteN,Varma S. An Unusual Presentation of Oral Squamous Cell Carcinoma:A Case Report.J Med Cases 2016;7(6):242-244

5. KaushalN,MadanN.Verrucous Carcinoma of the Oral Cavity:CaseReport.IJGG 2009;6(1):1-4

6. SadasivanA,ThankappanK,RajapurkarM,ShettyS,SreehariS,IyerS.Verrucous lesions of the oral cavity treated with surgery:Analysis of clinico-pathologic features and outcome.ContempClin Dent 2012;3:60-3

7. RekhaK.P,AngadiPV.Verrucous carcinoma of the oral cavity:aclinico-pathologic appraisal of 133 cases in Indians.OralMaxillofacSurg 2010;14:211-218

8. ShergillA.K,SolomonMC,CamelioS,KamathAT,AramanadkaC,ShergillG.S.Verrucous Carcinoma of the Oral Cavity:CurrentConcepts.Int J Sci Stud 2015;3(3):114-118

9. BhavirisettyD,ParvezA,NarsinganiS,QureshiMY.Verrucous Carcinoma of Oral Cavity-A Case Report and the Review of Literature.Asian Pac. J.HealthSci 2016;3(2):92-95

10. AgnihotriA,AgnihotriD.Verrucouscarcinoma:A study of 10 cases.Ind J Oral Sci 2012;3:79-83

11. VarshneyS,SinghJ,SaxenaRK,Kaushal A and PathakVP:Verrucous carcinoma of larynx.Indian J Otolaryngol Head Neck Surg;2004;56(1):54-56

12. Singh K,KalsotraP,Khajuria $R$ and Manhas M:Verrucous carcinoma(Ackerman's Tumour) of mobile tongue.JK Science 2004;6(4):220-222

13. VivekanandhRG,RamlalG,JitenderRK,RajshekarP.Proloferativeverrucousleukoplakia and verrucous carcinomaA Diagnostic dilemma-case report.Ann Essences Dent 2010;4:52-55

14. WarnakulasuriyaS.Global epidemiology of oral and oropharyngealcancer.Oral Oncol.2009;45(4-5):309-316

15. Thompson LD.Squamous cell carcinoma variants of the head and neck.CurrDiagnPathol 2003;9:384-96 